\title{
Construção e validação de instrumento para consulta de enfermagem à pacientes de grupos-alvo ou com doença renal crônica na atenção primária à saúde
}

\author{
Construction and validation of an instrument for nursing consultation for patients from target \\ groups or with chronic kidney disease in primary health care \\ Construcción y validación de un instrumento de consulta de enfermería para pacientes de grupos \\ objetivo o con enfermedad renal crónica en atención primaria de salud
}

Recebido: 03/09/2021 | Revisado: 10/09/2021 | Aceito: 14/09/2021 | Publicado: 16/09/2021

\author{
Welmer Danilo Rodrigues Rocha \\ ORCID: https://orcid.org/0000-0003-4495-2436 \\ Instituto Federal de Educação, Ciência e Tecnologia do Tocantins, Brasil \\ E-mail: welmerrocha@gmail.com \\ Nivaldo Antonio Parizotto \\ ORCID: https://orcid.org/0000-0003-1774-9053 \\ Universidade Federal da Paraíba, Brasil \\ E-mail: nivaldoaparizotto@hotmail.com
}

\begin{abstract}
Resumo
O estudo teve como objetivo construir e validar um instrumento para consulta de enfermagem à pacientes de gruposalvo ou com doença renal crônica na Atenção Primária à Saúde. Trata-se de uma pesquisa metodológica do tipo desenvolvimento de ferramentas. O estudo foi realizado em quatro fases: 1 . Construção do instrumento com base na revisão integrativa da literatura, 2. Concepção do instrumento que contemplou as etapas do processo de enfermagem, 3. Recrutamento dos juízes para participar do estudo e 4. Validação de conteúdo quanto a estrutura, apresentação, clareza e relevância do material junto a 18 juízes. Realizou-se a análise dos dados com base no Índice de Validação de Conteúdo (IVC) e o teste binomial para avaliar a concordância e consistência dos especialistas em relação aos itens do instrumento. Na análise estatística, o IVC global do instrumento foi de 0,90 (IC95\%: 0,86 - 0,93). Quando realizado o teste binomial para identificar concordância entre os avaliadores, observou-se que quando estipulada uma concordância de $80 \%$, não foi possível obter diferenças entre os juízes. Quanto à avaliação dos itens do instrumento de consulta de enfermagem, 94,4\% (n=17) dos juízes consideraram que todos os aspectos do histórico, diagnósticos de enfermagem e intervenções e resultados estavam claros e relevantes. Conclui-se que o estudo atingiu seus objetivos quanto a validação dos conteúdos como estrutura, apresentação, clareza e relevância do material. Verificou-se que todo conteúdo contido no instrumento foi considerado significativo para a melhoria da qualidade da consulta de enfermagem ao paciente de grupos-alvos ou com DRC.
\end{abstract}

Palavras-chave: Doença renal crônica; Tecnologia; Enfermagem; Validação.

\begin{abstract}
The study aimed to build and validate an instrument for nursing consultation to patients from target groups or with chronic kidney disease in Primary Health Care. This is a methodological research of the development of tools type. The study was carried out in four phases: 1 . Construction of the instrument based on an integrative literature review, 2. Conception of the instrument that covered the stages of the nursing process, 3 . Recruitment of judges to participate in the study, and 4. Content validation regarding the structure, presentation, clarity and relevance of the material with 18 judges. Data analysis was performed based on the Content Validation Index (CVI) and the binomial test to assess the experts' agreement and consistency in relation to the instrument's items. In the statistical analysis, the instrument's global CVI was 0.90 (95\%CI: $0.86-0.93$ ). When performing the binomial test to identify agreement between the evaluators, it was observed that when an agreement of $80 \%$ was stipulated, it was not possible to obtain differences between the judges. As for the evaluation of the items of the nursing consultation instrument, 94.4\% $(n=17)$ of the judges considered that all aspects of the history, nursing diagnoses and interventions and results were clear and relevant. It is concluded that the study achieved its objectives regarding the validation of contents such as structure, presentation, clarity and relevance of the material. It was found that all content contained in the instrument was considered significant for improving the quality of nursing consultations for patients from target groups or patients with CKD.
\end{abstract}

Keywords: Chronic kidney disease; Technology; Nursing; Validation. 


\begin{abstract}
Resumen
El estudio tuvo como objetivo construir y validar un instrumento de consulta de enfermería a pacientes de grupos objetivo o con enfermedad renal crónica en Atención Primaria de Salud, se trata de una investigación metodológica de tipo desarrollo de herramientas. El estudio se realizó en cuatro fases: 1. Construcción del instrumento a partir de una revisión integradora de la literatura, 2. Concepción del instrumento que abarcó las etapas del proceso de enfermería, 3. Reclutamiento de jueces para participar en el estudio, y 4 Validación de contenido en cuanto a estructura, presentación, claridad y relevancia del material con 18 jueces. El análisis de los datos se realizó con base en el Índice de Validación de Contenido (IVC) y la prueba binomial para evaluar la concordancia y consistencia de los expertos en relación a los ítems del instrumento. En el análisis estadístico, el CVI global del instrumento fue 0,90 (IC del 95\%: 0,86 - 0,93). Al realizar la prueba binomial para identificar concordancia entre los evaluadores, se observó que cuando se estipuló una concordancia del $80 \%$, no fue posible obtener diferencias entre los jueces. En cuanto a la evaluación de los ítems del instrumento de consulta de enfermería, el 94,4\% $(n=17)$ de los jueces consideró que todos los aspectos de la historia, diagnósticos e intervenciones de enfermería y resultados fueron claros y relevantes. Se concluye que el estudio logró sus objetivos en cuanto a la validación de contenidos como estructura, presentación, claridad y relevancia del material. Se encontró que todo el contenido del instrumento se consideró significativo para mejorar la calidad de las consultas de enfermería para pacientes de grupos objetivo con ERC.
\end{abstract}

Palabras clave: Enfermedad renal crónica; Tecnología; Enfermería; Validación.

\title{
1. Introdução
}

A doença renal crônica (DRC) é uma causa importante de morbidade e mortalidade global. Estudos do Global Burden of Disease de 2013, estima-se que 956.200 pessoas morreram de DRC, um aumento de 134\% em relação a 1990, um dos maiores aumentos entre as principais causas de morte. Além disso, mesmo nos estágios iniciais da DRC, o risco de eventos cardiovasculares fatais e não fatais atribuíveis diretamente à doença renal aumenta substancialmente. Atualmente estudos de projeção demostram que a DCR em adultos com idade igual ou superior a 30 anos evidencia um aumento na prevalência de 13,2\% (2007-2012) para 14,4\% em 2020 e para 16,7\% em 2030 nos Estados Unidos. Considerando somente indivíduos com idade 65 ou mais anos, a prevalência estimada diminuirá de 39,6\% para 36,4\% em 2020, e então aumentará para 37,8\% em 2030 (Hoerger et al., 2015).

A doença renal compreende a lesão renal e dano progressivo e irrecuperável da função dos rins. Atualmente a DRC é avaliada por meio da taxa de filtração glomerular (TFG) e divide-se em cinco estágios, classificada de acordo com o grau de função renal, indicada pelo TFG. O estadiamento da doença estabelecidos em estágio 1 refere-se a TFG >90 mL/min/1,73m², em grupos de risco para DRC, na presença de proteinúria ou hematúria glomerular ou alteração no exame de; estágio 2 entre 60 e 89; estágio 3a e 3b entre 30 e 59; estágio 4 entre 15 e 29; estágio 5 <15. (Kdigo, 2013).

Os enfermeiros tem um papel fundamental na atenção básica a saúde $(\mathrm{AB})$ e uma das suas responsabilidades é incentivar o autocuidado à saúde. A atuação na prevenção e progressão da DRC é de localizar e identificar os grupos de risco, bem como os pacientes com a doença instalada, nos quais a avaliação da função renal é imprescindível. (Travagim; Kusumota, 2009).

A atenção primária tem como princípio a natureza múltipla dos fatores de risco que envolve a doença, sendo necessária uma abordagem integral e interdisciplinar. É constante os pacientes considerados grupos-alvos para o desenvolvimento da doença ser primeiramente atendido pela atenção primaria, para que estes pacientes não sejam direcionados de maneira tardia para a terapia substitutiva renal, é fundamental que os profissionais da atenção primária estejam capacitados para o reconhecimento da enfermidade, identificando as complicações e doenças associadas, focada em uma assistência a interromper ou diminuir a perda da função renal (Bastos; Bastos, 2007).

O processo de produção do conhecimento na inovação tecnológica é formado pelo ciclo de ideias no âmbito da ciência, por meio de pesquisa, criatividade e experimentação, obtendo um produto ou processo de fator aplicável. A doença renal crônica vem se destacando como causa importante de morbidade, no entanto o uso de uma tecnologia leve, como acolhimento, o aconselhamento e a visita domiciliar são inquestionáveis seus benefícios para a prevenção (Saraceni, 2012). 
Os enfermeiros estão em melhor posição para avaliar o estado de saúde funcional e as necessidades tecnológicas, devido às suas interações estreitas com os pacientes. Outras colaborações inovadoras entre enfermeiros e engenheiros biomédicos são necessárias em várias formas para alavancar e impactar positivamente o atendimento ao paciente. $\mathrm{O}$ mundo em evolução centrado na tecnologia da assistência à saúde também exigirá que os enfermeiros e prestadores de serviços de linha de frente estejam equipados com conhecimentos técnicos e de saúde. Como resultado, os campos de tecnologia da informação em saúde, enfermagem e engenharia precisarão colaborar para o propósito de força de trabalho e desenvolvimento econômico.

Durante a coleta de dados realizada para pesquisa aos cuidados prestados à pessoa com DRC na atenção básica de saúde questiona-se: um instrumento de consulta de enfermagem, com base na sistematização de assistência de enfermagem, é válido quanto ao conteúdo e aparência para direcionar o enfermeiro na condução da consulta e no acompanhamento de gruposalvo ou a paciente com doença renal crônica na atenção primária à saúde? O objetivo central desde estudo é construir e validar um instrumento para consulta de enfermagem à pacientes de grupos-alvo ou com doença renal crônica na Atenção Primária à Saúde.

\section{Metodologia}

Trata-se de uma pesquisa metodológica que conforme Polit e Beck (2019) busca enfocar o desenvolvimento de ferramentas específicas, coleta de dados, investigação, organização e análise dos dados com o intuito de proporcionar a melhoria, confiabilidade e validade desses instrumentos.

O estudo foi constituído por etapas, a primeira buscou-se reunir evidências de produções científicas a respeito da consulta de enfermagem a pacientes com doença renal crônica atendidas na atenção primária. No DeCS (Descritores em Ciências da Saúde), encontrou-se os seguintes descritores: doença renal crônica, tecnologia, enfermagem e validação. A busca procedeu-se no mês de junho de 2020 e a delimitação temporal ocorreu nos últimos 6 anos de 2015 a 2021. Os bancos de dados utilizados foram o PUBMED/MEDILINE, CINAHL Complete EBSCO, SciVerse Scopus e do Caribe em Ciência da Saúde (LILACS).

Para etapa de construção do instrumento de consulta de enfermagem, foi composto de 5 (cinco) fases com base na sistematização da assistência de enfermagem - investigação, diagnóstico, planejamento, implementação e evolução/resultados.

Em seguida, foi realizado a etapa de recrutamento dos participantes e coleta de dados, o qual foram selecionados pela a técnica de amostragem intencional, em que o pesquisador seleciona os membros que irão participar por decisão própria, baseado em suas especificidades acerca do tema estudado (Polit; Beck, 2011). Além deste, foi utilizado seleção de juízes por meio da amostragem de rede ou "bola de neve" (snowball) sendo este um método utilizado quando a população consiste em pessoas com características distintas. Desta forma, ao encontrar um participante que se enquadra nos critérios de elegibilidade será solicitada a indicação de outros possíveis participantes (Polit; Cheryl, 2019).

Os participantes foram denominados de juízes, foi enviado pelo Google Forms o Termo de Consentimento Livre Esclarecido (TCLE), para que fosse registrado sua anuência e em seguida responderam o instrumento de validação da tecnologia para consulta de enfermagem a pacientes de grupos-alvos ou com DRC.

$\mathrm{Na}$ etapa de validação do instrumento para consulta de enfermagem, foram utilizados dois instrumentos para validação do instrumento de consulta de enfermagem. O primeiro é uma escala do tipo Likert, pela necessidade de identificar a qualidade do constructo, por ser amplamente empregada e possuir representatividade científica e demanda menos recursos e tempo (Lucian; Dornelas, 2014). As respostas do instrumento utilizada no estudo foram apresentadas sob a forma da escala de Likert em quatro pontos equivalentes, 1- Inadequado (I), 2- Parcialmente adequado (PA), 3- Adequado (A) e 4- Totalmente adequado (TA). Esta escala foi utilizada em três domínios: Objetivo (cinco itens), Estrutura e Apresentação (nove itens) e Relevância (quatro itens). 
Para avaliar cada item, utilizou-se o cálculo do Índice de Validade de Conteúdo (IVC). Para calcular o IVC dos itens, foi dividido o número total de juízes, que atribuíram escore de 3 ou 4 em uma escala ordinal de quatro pontos com significância de "adequado" a "totalmente adequado", pelo total de juízes que avaliaram o item (Neusa; Marina, 2011). Foi considerado aceitável o IVC com taxa mínima de 0,80 para adequação de objetivos, estrutura e relevância, uma vez que autores recomendam esse valor como aceitável para validações com mais de 6 juízes (Polit; Beck, 2019).

Também houve o cálculo do teste binomial para avaliar a concordância e consistência dos especialistas em relação aos itens do instrumento, sendo definido um valor igual ou superior a $80 \%$ para considerar o item adequado se o teste não apresentar significância estatística ( $p>0,05$ ) uma vez que a hipótese nula é a de concordância. (Lopes; Silva; Araújo, 2013).

$\mathrm{O}$ segundo instrumento foi para a avaliação dos itens do instrumento de consulta de enfermagem. Este era composto por 3 itens (sim, em parte e não) utilizados para avaliar a clareza e a relevância dos domínios Histórico, Diagnóstico de enfermagem e Intervenções e resultados. Para a análise deste foi utilizado estatística descritiva por meio de frequências simples e relativas.

Todas as análises estatísticas foram realizadas no software Stata 13.0.

Após a validação do instrumento da consulta de enfermagem pelos juízes, foi realizada a etapa de correção do instrumento da consulta de enfermagem, foi analisado as sugestões e críticas de modo a refinar o instrumento.

A pesquisa foi cadastrada e aprovada no Comitê de Ética e Pesquisa da Universidade Brasil sob o parecer número 4.599.353.

\section{Resultados}

O estudo contemplou 18 juízes e quanto as características, os avaliadores apresentavam idade mediana de 31 anos (IQR: 25 - 34), variando de 23 a 39 anos. O sexo predominante foi o feminino (94,4\%; $\mathrm{n}=17$ e todos eram graduados em enfermagem. A maior parte dos juízes apresentou tempo de formação variando entre um a cinco anos $(33,3 \%$; n=6) e com formação na área de atenção primária ou saúde pública $(33,3 \%$; n=6).

A maior parte dos juízes referiu trabalhar atualmente na assistência $(72,2 \% ; n=13)$ e unidades básicas de saúde $(72,2 \% ; \mathrm{n}=13)$. A função desempenhada pela maioria foi a de assistência direta ao paciente $(77,8 \% ; \mathrm{n}=14)$. O tempo de trabalho mostrou-se similar entre as categorias $<1$ ano (38,9\%; $n=7)$, entre um e cinco anos $(22,2 \% ; n=4)$ e de seis a dez anos $(33,3 \% ; n=6)$. A maioria dos participantes trabalha com paciente hipertenso, diabético ou com insuficiência renal $(94,4 \%$; n=17). Conforme se observa na Tabela 1, a seguir. 
Tabela 1: Características dos juízes.

\begin{tabular}{|c|c|c|}
\hline & $\mathrm{N}$ & $\%$ \\
\hline \multicolumn{3}{|l|}{ Sexo } \\
\hline Feminino & 17 & 94,4 \\
\hline Masculino & 1 & 5,6 \\
\hline \multicolumn{3}{|l|}{ Formação } \\
\hline Enfermagem & 18 & 100,0 \\
\hline \multicolumn{3}{|l|}{ Tempo de formação } \\
\hline$<1$ ano & 3 & 16,7 \\
\hline 1 a 5 anos & 6 & 33,3 \\
\hline 6 a 10 anos & 5 & 27,8 \\
\hline$>10$ anos & 4 & 22,2 \\
\hline \multicolumn{3}{|l|}{ Titulação } \\
\hline Residência ou Especialização & 15 & 79,9 \\
\hline Mestrado & 2 & 11,1 \\
\hline \multicolumn{3}{|l|}{ Área de formação } \\
\hline Atenção primária ou saúde pública & 6 & 33,3 \\
\hline Gestão & 2 & 11,1 \\
\hline Obstetrícia & 2 & 11,1 \\
\hline Outro & 3 & 16,7 \\
\hline Não informado & 5 & 27,8 \\
\hline \multicolumn{3}{|l|}{ Ocupação atual } \\
\hline Assistência & 13 & 72,2 \\
\hline Ensino & 2 & 11,1 \\
\hline Outra & 3 & 16,7 \\
\hline \multicolumn{3}{|l|}{ Local de trabalho } \\
\hline UBS & 13 & 72,2 \\
\hline Gestão & 2 & 11,1 \\
\hline IES & 2 & 11,1 \\
\hline Hospital & 1 & 5,6 \\
\hline \multicolumn{3}{|l|}{ Função } \\
\hline Assistência & 14 & 77,8 \\
\hline Coordenação & 2 & 11,1 \\
\hline Ensino & 2 & 11,1 \\
\hline \multicolumn{3}{|l|}{ Tempo de trabalho } \\
\hline$<1$ ano & 7 & 38,9 \\
\hline 1 a 5 anos & 4 & 22,2 \\
\hline 6 a 10 anos & 6 & 33,3 \\
\hline$>10$ anos & 1 & 5,6 \\
\hline \multicolumn{3}{|c|}{$\begin{array}{l}\text { Trabalha com paciente hipertenso, diabético ou com } \\
\text { insuficiência renal }\end{array}$} \\
\hline $\operatorname{Sim}$ & 17 & 94,4 \\
\hline Não & 1 & 5,6 \\
\hline
\end{tabular}

Legenda: UBS - unidade básica de saúde; IES - Instituição de Ensino Superior. Fonte: Autores.

A avaliar o IVC dos itens de avaliação do instrumento identificou-se que todos os itens foram considerados validados (IVC>0,80). Na análise do IVC individual, o item relacionado ao terceiro objetivo "O uso do instrumento aos paciente-alvos ou com doença renal crônica contribuirá na adesão ao tratamento" foi o que apresentou menor valor (IVC $=0,83)$, mesmo assim o valor estava dentro do ponto de corte considerado para validade (IVC>0,80). O instrumento como um todo apresentou um IVC geral de 0,90 (IC95\%: 0,86-0,93).

Quando realizado o teste binomial para identificar concordância entre os avaliadores, observou-se que quando estipulada uma concordância de $80 \%$, não foi possível obter diferenças entre os juízes. Assim, o conteúdo foi validado com pelo menos $80 \%$ de concordância entre os avaliadores. O resultado pode ser acompanhado a partir da Tabela 2 a seguir. 
Tabela 2: Validação do conteúdo do instrumento de consulta de enfermagem.

\begin{tabular}{|c|c|c|c|c|}
\hline \multirow[b]{2}{*}{ Item do instrumento } & \multirow[b]{2}{*}{ IVC } & \multicolumn{2}{|c|}{ IC95\% } & \multirow[b]{2}{*}{ Sig. } \\
\hline & & $\begin{array}{c}\text { Limite } \\
\text { Inferior }\end{array}$ & $\begin{array}{c}\text { Limite } \\
\text { Superior }\end{array}$ & \\
\hline
\end{tabular}

\section{Bloco objetivos}

1. A linguagem utilizada está compatível com o público-alvo

2. O instrumento poderá promover mudança de comportamento e atitudes dos paciente-alvos a doença renal crônica

$0,94-0,73 \quad 1,00 \quad 0,10$

3. O uso do instrumento aos paciente-alvos a doença renal crônica contribuirá na adesão ao tratamento

$0,89 \quad 0,65 \quad 0,99 \quad 0,27$

4. O uso do instrumento facilitará o atendimento paciente-alvos a doença renal crônica

$0,83 \quad 0,59 \quad 0,96 \quad 0,50$

Bloco estrutura e apresentação

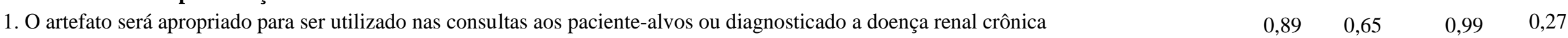

2. As informações são suficientes para realizar as etapas do Processo de Enfermagem

3. Há uma sequência lógica no instrumento

4. As informações apresentadas no instrumento estão cientificamente corretas

5. A organização do instrumento está adequada

6. O tamanho das letras dos títulos, subtítulos e texto está adequado

7. Os diagnósticos presentes no instrumento são compatíveis com a doença renal aguda/crônica

$0,94 \quad 0,73 \quad 1,00 \quad 0,10$

8. As intervenções apresentadas no instrumento são compatíveis com a doença renal aguda/crônica

$0,89 \quad 0,65 \quad 0,99 \quad 0,27$

9. Os resultados esperados no instrumento são compatíveis com a doença renal aguda/crônica

$0,89 \quad 0,65 \quad 0,99 \quad 0,27$

$0,89 \quad 0,65 \quad 0,99 \quad 0,27$

\section{Bloco relevância}

1. O instrumento para consulta de enfermagem irá servir como guia ao atendimento de paciente-alvos ou diagnosticado a doença renal crônica

2. O instrumento é necessário para o atendimento de paciente-alvos ou diagnosticado a doença renal crônica

$0,89 \quad 0,65 \quad 0,99 \quad 0,27$

3. O instrumento para consulta de enfermagem utilizado poderá trazer respaldo clínico ao enfermeiro

$0,89 \quad 0,65 \quad 0,99 \quad 0,27$

IVC geral do instrumento

\begin{tabular}{cccc}
0,89 & 0,65 & 0,99 & 0,27 \\
0,89 & 0,65 & 0,99 & 0,27 \\
0,89 & 0,65 & 0,99 & 0,27 \\
\hline 0,90 & 0,86 & 0,93 & -
\end{tabular}

Fonte: Autores. 
Quanto à avaliação dos itens do instrumento de consulta de enfermagem, 94,4\% (n=17) dos juízes consideraram que todos os aspectos do histórico, diagnósticos de enfermagem e intervenções e resultados estavam claros e relevantes. Somente um avaliador apontou como "em parte".

Somente uma sugestão foi dada quanto à clareza e somente para o diagnóstico. Sugeriu-se incluir a etapa planejamento após o diagnóstico e recursos físicos.

Quanto à relevância do histórico, foi sugerida a inclusão de espaço para inserção de histórico de doenças, fatores hereditários, medicações em uso. Acerca da relevância do diagnóstico, foi sugerida a inclusão a etapa planejamento após a etapa de diagnóstico (graduando o tempo esperado para o alcance da meta exemplo: 1 semana, 2 semanas, entre outros). Com relação à relevância das intervenções, foi sugerida inclusão da graduação alvo do resultado de 1 a 5, como proposto no NIC e NOC. Os resultados podem ser observados a partir da Tabela 3 abaixo.

Tabela 3. Avaliação da clareza e relevância dos componentes do instrumento de consulta de enfermagem.

\begin{tabular}{lccccc}
\multicolumn{1}{c}{ Itens do } & \multicolumn{2}{c}{ Clareza } & \multicolumn{2}{c}{ Relevância } & \multirow{2}{*}{ IVC } \\
\hline Instrumento & $\mathrm{N}$ & $\%$ & $\mathrm{~N}$ & $\%$ & \multirow{2}{*}{1} \\
Sistórico & 17 & 94,4 & 17 & 94,4 & \\
Em parte & 1 & 5,6 & 1 & 5,6 & \\
Não & 0 & 0 & 0 & 0 & 1 \\
Diagnósticos de Enfermagem & & & & \\
Sim & 17 & 94,4 & 17 & 94,4 & \\
Em Parte & 1 & 5,6 & 1 & 5,6 & \\
Não & 0 & 0 & 0 & 0 & 1 \\
Intervenções e resultados & 17 & 94,4 & 17 & 94,4 & \\
Sim & 1 & 5,6 & 1 & 5,6 & \\
Em Parte & 0 & 0 & 0 & 0 & \\
Não & & & & & \\
\hline
\end{tabular}

Fonte: Autores.

\section{Discussão}

Quanto à avaliação dos itens do instrumento de consulta de enfermagem, 94,4\% (n=17) dos juízes consideraram que todos os aspectos do histórico, diagnósticos de enfermagem e intervenções e resultados estavam claros e relevantes. Somente um avaliador apontou como "em parte".

Através dos registros foi possível observar que: $94 \%$ dos participantes eram do sexo feminino, faixa etária de 25-34 anos e que trabalham atualmente na assistência e unidades básicas de saúde. Esses dados se assemelham aos do $1^{\circ}$ Censo de Recursos Humanos da Atenção Primária em Minas Gerais, em 2006, que registrou 55.801 profissionais atuando na APS, naquele estado, prevalecendo o sexo masculino na categoria médica e o feminino na de enfermeiros, e, em relação à faixa etária, a maioria dos médicos e enfermeiros tinham idade entre 30-49 anos (Barbosa et Rodrigues, 2006).

Interessante observar que o tempo de formação da maioria dos enfermeiros, mostrou-se similar entre as categorias <1 ano $(38,9 \% ; n=7)$, entre um e cinco $\operatorname{anos}(22,2 \% ; n=4)$ e de seis a dez anos $(33,3 \%$; $n=6)$, o que demonstra que eram profissionais que atuavam na maior parte dos anos de trabalho, após a formação, na APS, ou seja, detinham uma relativa experiência e estabilidade em relação a esse ponto de atenção. Também é relevante o volume de profissionais que após a formação de graduação se inseriram em programas diversos de pós-graduação, refletindo um investimento educacional desses profissionais. Em estudo conduzido pelo Observatório de Recursos Humanos da Secretaria Estadual de Saúde SES-SP em 2008, com amostra representativa de 250 municípios do Estado, verificou-se que 45,9\% dos médicos que atuavam na $\mathrm{AB}$ Tradicional e 65,9\% no PSF não tinham nenhuma formação em residência médica. No estudo citado, 32,6\% dos médicos não 
possuíam essa formação e, outro fator importante, foi o predomínio da Residência em Medicina de Família e Comunidade. Esses achados podem ser reflexo de mudanças ocorridas nos últimos anos como o Programa de Valorização do Profissional da Atenção Básica - PROVAB (2011) e o Programa Mais Médicos (2013), iniciativas que destacaram a necessidade de formação com enfoque na APS.

Para o desenvolvimento e sustentabilidade do SUS o processo de formação profissional de saúde é essencial, pois o trabalho em saúde se baseia no elemento humano, ou seja, na sua capacidade de agir e refletir, de compreender os determinantes do processo saúde-doença em sua dinamicidade e complexidade e em sua empatia (Campos et al., 2008). Assim, a formação na graduação, pós-graduação e os processos de educação permanente nos serviços constituem esse processo continuamente. A OPAS (2015) aponta a escassez mundial de trabalhadores de saúde qualificados para atender as diversas necessidades de saúde da população em geral e, além disso, que a maior parte da força de trabalho atual foi treinada para tratar de enfermidades agudas, e não de problemas crônicos, que exigem diferentes competências e habilidades.

Apesar de menos da metade dos médicos terem recebido algum tipo de treinamento, na maioria das vezes, esses foram ofertados pelos serviços em que atuavam, enquanto que para os enfermeiros observou-se uma escassez de oferta de treinamento em DRC e ainda um expressivo número de busca de treinamentos por meios próprios, o que sugere a não priorização dessa categoria profissional para os treinamentos nessa temática.

Em paralelo, um estudo realizado em Juiz de Fora município de Minas Gerais, o qual avaliou a estrutura, processo e resultado do "Programa de Atenção a Doentes Renais" no município, observou-se que a maior parte dos profissionais não recebeu capacitação específica para o atendimento de usuários com DRC. O baixo percentual de profissionais com perfil e capacidade técnica qualificada estão entre os obstáculos citados pelos gestores da atenção primária que colabora para baixa fixação dos profissionais e, consequentemente, para a fragmentação da rede (Paula et al., 2016).

A maior parte dos juízes referiu trabalhar atualmente na assistência e unidades básicas de saúde $(72,2 \%$; n=13). A função desempenhada pela maioria foi a de assistência direta ao paciente $(77,8 \%$; n=14). Conforme, Monteiro e Hora (2014) é imprescindível a validação de uma tecnologia com profissionais especialistas e que tenham qualificação no conteúdo de interesse.

Nessa premissa, no atual trabalho optou-se por escolher profissionais com expertises que apresentassem desejável experiência na área, pois o julgamento e apreciação de profissionais especializados e envolvidos com o público alvo, proporcionam em pareceres e contribuições tanto no conteúdo quanto aparência, consoante recomendado por Moreira, Nóbrega e Silva (2003).

Com base nos achados acima destaca-se que foi de suma importância a participação destes enfermeiros assistenciais, com experiência profissional na área da atenção primária, pois trazem uma riqueza de saberes e conhecimentos específicos para contribuir com o instrumento, além de uma prática já embasada em evidências.

É relevante ressaltar, que ao avaliar o IVC dos itens de avaliação do instrumento, identificou-se que todos os itens foram considerados validados (IVC>0,80), e que o instrumento como um todo apresentou um IVC geral de 0,90 (IC95\%: 0,86 - 0,93). Em relação ao IVC Alexandre e Coluci (2011) compreendem como o método muito utilizado na área da Saúde, que permite saber a proporção ou percentagem da concordância entre os juízes, desta forma, obtivemos um IVC total do instrumento de 0,90 , o que indica sua validade.

É importante considerar que no processo de educação em saúde o usuário é um elemento essencial para validação de um instrumento (ECHER, 2005). Isto posto, Doak, Doak e Root (1996) ressalta a relevância do processo de validação de material com população leiga, visto que serão esses usuários que irão usufruir deste material, assim como, em outros estudos foram procedidos a validação com o público-alvo (Mota, 2019; Balsells, 2018). 
Para tanto, essa etapa teve como propósito a validação pertinente ao objetivo, estrutura, apresentação, clareza e relevância deste instrumento. Destarte, os juízes avaliaram positivamente o instrumento, nós como pesquisadores consideramos imprescindível essa etapa para validação e adequação deste instrumento, pois através dos depoimentos de alguns participantes pode-se constatar a relevância do material.

Destacam-se algumas sugestões que foram acatadas e aquelas não acatadas, para elaboração do instrumento final de consulta de enfermagem.

Dentre as sugestões não acatadas, ressalta-se no item quanto à clareza somente uma sugestão foi dada para o diagnóstico. Sugeriu-se incluir a etapa planejamento após o diagnóstico e recursos físicos.

Conforme Brasil (2017) planejamento de enfermagem constitui também as ações ou intervenções de enfermagem que serão realizadas face às respostas da pessoa, família ou coletividade humana em um dado momento do processo saúde e doença, identificadas na etapa de Diagnóstico de Enfermagem. A etapa planejamento não se encontra explicita, entretanto ela atua no instrumento.

Com objetivo em construir um instrumento completo, em que vislumbre o ser humano como um ser indivisível e único, e sabendo da alta demanda de pacientes e dos cuidados assistenciais na atenção primária, bem como do número de enfermeiros nem sempre condizente para a realização de todas estas atividades, atribui-se elaborar um instrumento com apresentação sucinta e objetiva. Lembrando que no Art. 71 das Responsabilidades e Deveres do Código de Ética de Enfermagem temos que incentivar e criar condições para registrar as informações inerentes e indispensáveis ao processo de cuidar (Brasil, 2017).

O conteúdo e a dinâmica do instrumento abordaram os diagnósticos, intervenções e resultados de enfermagem construídos e planejados especificamente para as necessidades de cuidados de cada paciente alvo ou com doença renal crônica previamente, além de exprimir as intervenções e os resultados das condutas terapêuticas preconizadas por enfermeiros no planejamento da assistência de enfermagem.

Ao elaborar um instrumento com uma teoria interligada tem-se o respaldo e a firmeza de se obter e alinhar os resultados esperados e sistematizados a partir de estudos e validações já realizados, com maior credibilidade e segurança. De tal modo a sugestão não foi acatada.

Outra, sugestão não acatada, foi acerca da relevância do diagnóstico, o qual sugeriu-se a inclusão da etapa planejamento após a etapa de diagnóstico (graduando o tempo esperado para o alcance da meta exemplo: 1 semana, 2 semanas, entre outros).

A listagem dos possíveis diagnósticos de enfermagem segundo a taxonomia da NANDA I visou compatibilizar layout; rapidez no preenchimento, acompanhamento processual da evolução dos diagnósticos e explicitação de todos os componentes de cada diagnóstico (causas, fatores de risco e manifestações clínicas) e nortearam, simultaneamente, a escolha das intervenções de enfermagem e a explicitação dos indicadores de resultados utilizados na evolução de enfermagem, contribuindo para a melhoria dos registros de enfermagem (Herdman, 2015).

Conciliar as prioridades de demandas de cuidados e necessidades emergentes com a organização dos conteúdos, foi critério para apresentação dos diagnósticos de enfermagem. A experiência de adequar os diagnósticos buscou atender à recomendação do processo de sistematização da assistência enfermagem para facilitar o manuseio do instrumento pelos enfermeiros. Como já descrito a etapa planejamento encontra-se após a etapa diagnóstico. Em referência ao alcance das metas, os diagnósticos estão determinados para registros diário com base na evolução dos diagnósticos de enfermagem por meio de códigos que indicarão o início, o acompanhamento ou o término de um diagnóstico. Assim a sugestão não foi acatada.

Com relação à relevância das intervenções, foi sugerida inclusão da graduação alvo do resultado de 1 a 5 , como proposto no NIC e NOC. 
As intervenções e os resultados de enfermagem, segundo a NIC e a NOC, foram agrupados em um mesmo impresso para possibilitar: compatibilizar um layout e otimizar espaço; dar rapidez ao preenchimento do registro; facilitar o manuseio e ligar as intervenções aos indicadores de resultados (escalas mensurativas), com espaço para aprazamento dos mesmos. O critério adotado foi a garantia da explicitação das diretrizes gerais para se definir a conduta terapêutica, os indicadores e as escalas mensurativas para cada item.

As escalas contêm valores que variam de um e cinco, significando a melhor condição do paciente o valor cinco. Deste modo a sugestão não foi acatada por já existir a graduação alvo no instrumento.

Ao que concerne a sugestão acatada, foi considerado pertinente, sendo realizada a modificação no instrumento. Destaca-se aqui a sugestão para inclusão de espaço para inserção de histórico de doença, fatores hereditários e medicações em uso no item histórico de enfermagem. Assim, foram revistas as páginas que apresentaram layout com descontinuidade de conteúdo, para possibilitar a inserção desse novo item.

Após a avaliação do público-alvo, a versão final do instrumento foi composta por 6 páginas, sendo 2 páginas destinadas ao histórico de enfermagem, 2 páginas aos diagnósticos de enfermagem e 2 páginas as intervenções e resultados de enfermagem.

\section{Conclusão}

A vivência de elaborar e validar um instrumento de consulta de enfermagem possibilitou a compreensão da importância destes dados para uma assistência de qualidade destes pacientes alvos ou com doença renal crônica, no sentido de se elaborar um plano de cuidados mais individualizado e pertinente às reais necessidades do paciente.

A seleção dos juízes especialistas, com experiência na área assistencial, bem como de ensino e de pesquisa, bem como o embasamento teórico e os estudos já citados permitiram a elaboração deste instrumento. Acredita-se na importância da SAE e de realizar a suas etapas da sistematização de forma completa, abordando o paciente como um todo.

Apesar do item de avaliação relacionado ao terceiro objetivo "O uso do instrumento aos paciente-alvos ou com doença renal crônica contribuirá na adesão ao tratamento" ter apresentado menor valor (IVC $=0,83$ ), mesmo assim o valor estava dentro do ponto de corte considerado para validade (IVC $>0,80$ ). Contudo, o instrumento como um todo apresentou um IVC geral de 0,90 (IC95\%: 0,86 - 0,93), nos mostrando que o instrumento é válido e será importante para um cuidado mais humanizado e individualizado.

Todavia espera-se que este instrumento venha a fazer a diferença no cuidar do enfermeiro dando subsídios para uma coleta de dados mais detalhada e direcionada do paciente visando uma assistência de qualidade e excelência para o paciente.

Diante das adversidades do estudo, destaca-se como limitações o baixo número de profissionais de enfermagem especializado em nefrologia, principalmente com atuação na atenção primária a saúde. Outro ponto observado é que o conhecimento sobre doença renal crônica entre os profissionais de enfermagem ainda é restrito, identificando uma necessidade de educação permanente. Porém, os que contribuíram para o aprimoramento da tecnologia, agregaram conhecimentos e valores à sua versão final.

Sugere-se, contudo, que novos estudos sejam realizados, buscando-se verificar a confiabilidade e eficiência do instrumento a partir do autorrelato dos profissionais, em virtude da conveniência de conferir os resultados alcançados a partir de uma metodologia observacional.

\section{Referências}

Alexandre, N. M. C. \& Coluci, M. Z. O. (2011). Validade de conteúdo nos processos de construção e adaptação de instrumentos de medidas. Ciência \& Saúde Coletiva https://doi.org/10.1590/S1413-81232011000800006 
Barbosa, A. C. Q. \& Rodrigues, J. M. (2006). Primeiro censo de recursos humanos da atenção primária do Estado de Minas Gerais. Belo Horizonte: Observatório de Recursos Humanos em Saúde, UFMG,

Bastos, R. M. R. \& Bastos, M. G. (2007). Inserção do programa de saúde da família na prevenção da doença renal crônica. J. Bras. Nefrol., 29 , $32-34$.

Campos, G. W. S., Guerrero, A. V. P. (2008). Manual de práticas de atenção básica: saúde ampliada e compartilhada. Aderaldo \& Rothschild.

Brasil. (2009). Conselho Federal de Enfermagem (BR). Resolução No 358 do Conselho Federal de Enfermagem, de 15 de outubro de 2009 (BR). Dispõe sobre a Sistematização da Assistência de Enfermagem e a implementação do Processo de Enfermagem em ambientes, públicos ou privados, em que ocorre o cuidado profissional de Enfermagem, e dá outras providências. http://www.cofen.gov.br/resoluo-cofen-3582009_4384.html

Brasil. (2017). Conselho Federal de Enfermagem (COFEN). Resolução COFEN nº 564, de 6 de novembro de 2017. Aprova a reformulação do Código de Ética dos profissionais de enfermagem. http://www.cofen.gov.br/resolucao-cofen-no-5642017_59145.html

Doak, C. C., Doak, L. G., \& Root, J. H. Teaching patients with low literacy skills. (2a ed.), Lippincott, 1996.

Echer, I. C. Elaboração de manuais de orientação para o cuidado em saúde. Revista Latino-Americana de Enfermagem. 2005. https://doi.org/10.1590/S010411692005000500022

Herdman, T. H. \& Kamitsuru, S. (2017). NANDA International nursing diagnoses: Definitions classification, 2018-2020. Theime, http://dx.doi.org/10.1055/b006-161141

Hoerger, T. J., Simpson S. A., Yarnoff B. O., Pavkov M. E., Burrows N. R., Saydah S. H., Williams D. E., \& Zhuo X, (2015). The future burden of CKD in the United States: a simulation model for the CDC CKD Initiative. American Journal of Kidney Diseases, 65, 403-411...

Kidney Disease: Improving Global Outcomes (KDIGO) CKD Work Group. Leitura rápida do KDIGO 2012: Diretrizes para avaliação e manuseio da doença renal crônica na prática clínica. Kidney Int (Suppl) 2013, 3:1-150. https://www.scielo.br/pdf/jbn/v36n1/0101-2800-jbn-36-01-0063.pdf.

Lopes, M. V. O., Silva, V. M., \& Araújo, T. L. (2013). Validation of nursing diagnosis: challenges and alternatives. Rev Bras Enferm. 66, 649-55, http://dx.doi.org/10.1590/S0034- 71672013000500002. https://www.scielo.br/scielo.php?pid=S0034-71672013000500002\&script=sci_abstract

Lucian, R., \& Dornelas, J. S. (2015). Mensuração de Atitude: Proposição de um Protocolo de Elaboração de Escalas. Revista de Administração Contemporânea, 19, 157-177.

Monteiro, G. T. R., \& Hora, H. R. M. (2014). Pesquisa em saúde pública: como desenvolver e validar instrumentos de coleta de dados. Curitiba: Appris,.

Moreira, M. F., Nóbrega, M. M. L. \& Silva, M. I. T. (2003). Comunicação escrita: contribuição para a elaboração de material educativo em saúde. Revista Brasileira de Enfermagem. https://doi.org/10.1590/S0034-71672003000200015

Oliveira, L. L., Mendes, I. C., Balsells, M. M. D., Bernardo, E. B. R., Castro, R. C. M. B., Aquino, P. S. \& Damasceno, A. K. C. (2019). Educational hypermedia in nursing assistance at birth: building and validation of content and appearance. Rev Bras Enferm. https://doi.org/10.1590/0034-7167/2018-0163

Organização Pan-Americana da Saúde. (2015). Plano de ação sobre a saúde dos trabalhadores. 54 Conselho Diretor da OPAS, 67 ${ }^{a}$ Sessão do Comitê Regional da OMS para as Américas, de 28 de setembro a 2 de outubro de 2015, Washington, DC. Washington, DC: OPAS.

Paula, E. A., Costa, M. B., Colugnati, F. A. B., Bastos, R. M. R., Vanelli, C. P., Leite, C. C. A., Caminhas, M. S., \& Paula R. B. (2016). Strengths of primary healthcare regarding care provided for chronic kidney disease. Rev. Latino-Am. Enfermagem. http://dx.doi.org/10.1590/1518-8345.1234.2801.

Polit, D. F. \& Beck, C. T. (2019). Fundamentos de pesquisa em enfermagem: avaliação de evidências para a prática da enfermagem. (9a ed.), p.196, Artmed.

Saraceni, V., \& Miranda, A. E. (2012). Relação entre a cobertura da Estratégia Saúde da Família e o diagnóstico de sífilis na gestação e sífilis congênita. Cadernos de Saúde Pública. 28, 490-496. https://doi.org/10.1590/S0102-311X2012000300009

Seixas, P. (2009). Avanços e perspectivas na política de gestão de recursos humanos: desenvolvimento de pessoas e qualificação dos profissionais do SUS/SP. BIS, Bol. Inst. Saúde (Impr.) 48, 15-25 <http://periodicos.ses.sp.bvs.br/scielo.php?script=sci_arttext\&pid=S1518-18122009000300004\&lng=pt\&nrm=iso

Travagim, D. A. S., \& Kusumota, L. (2009). Atuação do enfermeiro na prevenção e progressão da doença renal crônica. Rev Enferm UERJ., $17,388-93$.

Wynd, C. A., Schmidt B., \& Schaefer, M. A. (2003). Two quantitative approaches for estimating content validity. West J Nurs Res. https://pubmed.ncbi.nlm.nih.gov/12955968/ 